\title{
Reduction of Fermat's Last Theorem in the Case that $A B C$ is Divisible by $n$ and Other Theorems
}

\author{
Spyridon Stamatopoulos, School Advisor, 27 Alkinoou St., 26442 Patras P.I.
}

tel.2610425751,6977748169, email: spyrosstamatopoulos891@gmail.com

Received: May 02, 2016 / Accepted: May 30, 2016 / Published: July 25, 2016.

\begin{abstract}
Proof of difficult mathematical problems primarily interests mathematicians. In other disciplines, there is great interest in the reasoning used, the cause of errors and the usefulness of conclusions. During the progress and development of a discipline - in our case Mathematics - it is essential for complex or simple assignments to be classified under one heading and referred to by a name. This results in their returning after some time to the desktop with reduced importance. Therefore, the use of an appropriate and widely understood language that does not alter the content is required, so that the knowledge is disseminated for use by whatsoever means (oral or written speech and the internet). Proof of a difficult problem that has troubled mathematicians for centuries is a significant event for mathematicians and worthy of investigation, while other disciplines are interested in the jurisprudence of arguments and the reasons for delayed progress of any effort. Thus, Philosophy can record the impacts of the mental environment formed by the prevailing intellectual activity of an era; Sociology can draw its own conclusions and the Applied Sciences can justify and decide on a new status for their field. A key factor in the understanding and processing of a problem is the language used (set theory, Spyros K. Kanellidis). During the progress and development of a discipline - in our case Mathematics - it is essential for complex or simple assignments to be classified under one heading and referred to by a name. This results in their returning after some time to the desktop with reduced importance. However, the excessive specialized use of scientific knowledge using advanced terminology and prevalence of the use of previous proven methods inhibit free and creative work (Werner Heisenberg: Encounters with Einstein). Indeed, the contemporary use of computers aggravates or constrains initiative, unless used for purposes of investigation, e.g. for numerical, graphical and perhaps bibliographic questions. Consequently, the use of terms that directly reveal what they define is required, e.g. median intersection and not centroid, or there should be description, e.g. the number 0.99999 (infinite number of 9s) very closely approaches number 1 and not lim0,9999 .... = 1
\end{abstract}

Examples of misinterpretation and false conclusions.

A. Determine the remainder of division $\left(\chi^{2}+1\right):(\chi+1)$ (T. N. Kazantzis, Number Theory, problem 50, p. 113).

Compute the remainder of division $\left(\chi^{2}+1\right):(\chi+1)$ for

all values of $\chi \in \mathrm{N}$

I apply the remainder theory as in algebra.

I set $\mathrm{f}(\chi)=\chi^{2}+1$ and $\delta(\chi)=\chi+1=\chi+\mathrm{c} \rightarrow \mathrm{v}=$ $\mathrm{f}(-\mathrm{c})$, so $\mathrm{v}=(-1)^{2}+1=2$.

I apply the integer division theory.

Since $\chi$ is an integer, $\chi^{2}+1$ and $\chi+1$ are also integers and therefore we have integers supposing $\pi(\chi)$ $=\chi^{+} \rho$ is the quotient and $v$ is the remainder.
In any case, $\chi+1>0$ (if $\chi+1<0$ we change the signs $\left.\frac{\Delta}{\delta}=\frac{-\Delta}{-\delta}\right), \chi \neq-\mathbf{1}$ and $0 \leq v<\chi+1$

Is $\chi^{2}+1=(\chi+\rho)(\chi+1)+v \rightarrow \chi^{2}+1=\chi^{2}+\chi$ $+\rho \chi+\rho+v \rightarrow \rightarrow(\rho+1) \chi=-(\rho+1)-v+2$. (1a) If $\rho+1 \neq 0 \rightarrow \chi=-1-\frac{v-2}{\rho+1}$. (1)

Since $\chi$ is an integer $\rho+1$ must divide the $v-2$, so $\rho$ $+1 \leq v-2 \rightarrow \rightarrow \rho+1<\chi+1-2 \rightarrow \rho<\chi-2$ and $\chi>\rho+$ 2. (2)

If $v=0, \rho+1$ divides $v-2 \rightarrow \rho+1= \pm 1$, or $\rho+1=$ $\pm 2$

from $(1) \rightarrow \chi=-1 \pm 2 \rightarrow \chi=1$, or $\chi=-3$, or $\chi=-1$ \pm 1 , or $\chi=0$, or $\chi=-2$ If $\rho+1=0$ from (1a) $\rightarrow v=2$ when $\chi<-3$ or $\chi>1$ The remainders in algebraic theory and 
number theory in the interval $-3 \leq \mathrm{x} \leq 1$ differ.

Question: Are there values of $\mathrm{x}$ such that the remainder is other than 0 and 2 ?

i) I apply the form $\mathrm{f}(\mathrm{x})=\mathrm{q}(\mathrm{x})(\mathrm{x}+\mathrm{c})+\mathrm{r}$ (Euclidean division in algebra) used in algebra with $\mathrm{x}+\mathrm{c}=0$, resulting in the form of the remainders $r=f(-c)$ so $r=2$. ii) The remainder in number theory If $v>2$ from (2) $\rightarrow \chi>\rho+2$, or

$$
\begin{aligned}
& \rho+2<\chi=-1-\frac{v-2}{\rho+1}, \text { from }(1), \rightarrow \rho+2<-1- \\
& \frac{v-2}{\rho+1} \rightarrow \rho+3<\frac{2-v}{\rho+1} \text {. (3) If } \rho+1>0 \rightarrow(\rho+3)(\rho+1) \\
& <2-v \rightarrow \rho^{2}+4 \rho+1+v<0 .
\end{aligned}
$$

The discriminant $\Delta=4^{2}-4(1+v)=12-4 v=4$ (3-v) when $v>2$ is negative or 0 , so the left-hand side of the inequality is always positive and the inequality has no solution . otherwise:

(If $\rho+1>0 \rightarrow \rho+3>0$, and since $v>2 \rightarrow 2-v<0$, so from (3) the positive $\rho+3$ is less than the negative $\frac{2-v}{\rho+1}$ which is not true.)

The residue thus be 0 or 1 , or 2 .

If $v=1 \rightarrow$ from $(1) \rho+1=+1 \rightarrow \delta(\chi)=\chi+1=1=$ $v$, rejected, or

$$
p+1=-1 \rightarrow \chi+1=-1 \rightarrow \delta(\chi)=|\chi+1|=1=v
$$
rejected.

Thus, the remainder in $\mathrm{N}$ is 0 or 2 . Generally, the remainder $v(\chi)$ does not give the numerical values of the remainder of the division, but gives the properties of the remainder - the body to which it belongs The correct definition of $v=\mathrm{f}(-\mathrm{c})$ is as follows: $\mathrm{v}=\mathrm{f}(-\mathrm{c})$ is a barrier, which partitions the domain of the function $\chi$ $\rightarrow \mathrm{r}(\chi)$ through the $\mathrm{f}(\chi):(\chi+\mathrm{c})$ in a constant function $\chi \rightarrow \mathrm{f}(-\mathrm{c})$, when $\chi \geq \mathrm{f}(-\mathrm{c})-\mathrm{c}$, and in one other $\chi \rightarrow \rightarrow$ $v(\chi)$ through the $v(\chi):(\chi+c)$, when $\chi<f(-c)-c$ This means that the remainder theory of the division of integer polynomials is not compatible with the integer division theory (Euclidean division). The proper process to find the remainder with $\chi+\mathrm{c}$ as divisor is to put the dividend $\mathrm{f}(\chi)$ in the form $\mathrm{f}(\chi)=\pi(\chi)(\chi+\mathrm{c})+$ $v^{\prime}\left(\right.$ Robert B. Ash Algebra p13,Capter2) - if $\boldsymbol{v}^{\prime}>\chi^{+c}$ fot same $\chi \rightarrow \boldsymbol{v}^{\prime}=\boldsymbol{\pi}^{\prime}(\chi)(\chi+\boldsymbol{c})+\boldsymbol{v}^{\prime \prime}$ ect - and to find the range of $v^{\prime}$, supposing that $\mathrm{f}\left(\chi_{\mathrm{i}}\right)$, then the remainder of $\mathrm{f}(\chi)$ with divisor $\chi+\mathrm{c}$ is the remainder of $v^{\prime}$ with divisor $\chi+c$, as the remainder of $\pi(\chi)(\chi+c)$ with $\chi+c$ is 0 .

Therefore: 1) if the value of $v^{\prime}<$ the value of $\chi+c$, for some $\chi$ (the degree of $v^{\prime}$ is always less than the degree of $\chi+c)$, then $v^{\prime}$ is the remainder.

2) If the value of $v^{\prime}>$ the value of $\chi+c$, then the remainder $v$ is the remainder of $v^{\prime}$, when divided by $\chi+\mathrm{c}$.

Note: In any case, $\chi+c>0$. If $\chi+c<0$, then we change the signs in $\mathrm{f}(\chi)$ and $\chi+\mathrm{c},\left(\frac{\Delta}{\delta}=\frac{-\Delta}{-\delta}\right)$. see Theorem (1) In the case where $v=\mathrm{f}\left(\chi_{\mathrm{i}}\right)<0$, supposing $\mathrm{f}\left(\chi_{\mathrm{i}}\right)=-\mathrm{k}\left(\chi_{\mathrm{i}}+\mathrm{c}\right)-\mathrm{v}, 0<\mathrm{v} \leq \chi_{\mathrm{i}}+\mathrm{c}$, then $\mathrm{f}\left(\chi_{\mathrm{i}}\right)=\chi-(\mathrm{k}+$ 1) $\left(\chi_{i}+c\right)+\left(\chi_{i}+c-r\right)$, and the remainder is $\left(\chi_{i}+c-v\right)$. see Theorem (1)

It is ascertained here that during the extension of a set, in our example the ring $\mathrm{N}$ is extended to $\mathrm{R}$, the operations defined in the new extended body can correspond only to one RESTRICTION in the initial body. (Kappos, p.58, Calculus1962).

B. When is the trinomial $x^{2}+b x+c$ a perfect square?

The answer given is when $\mathrm{D}=0$

The correct question is: When is the trinomial $\mathrm{x}^{2}+$ $\mathrm{bx}+\mathrm{c}$ a perfect square of an integer polynomial? Or we determine for what values of $x$ the trinomial takes square values.

In the first case, the answer is when $\mathrm{D}=0$ and,

in the second case, the values found for $\mathrm{x}$ and $\mathrm{d}$ from the solution of the Diophantine equation of first degree $x^{2}+b x+c=(x+d)^{2}$.

(Theorem: The trinomial $x^{2}+b x+c$ is a perfect square integer only when there are two divisors of the discriminant $b^{2}-4 c$, namely $d_{1}, d_{2}$, with a difference being a multiple of 4 and $d_{1} d_{2}=D$ ). see theorem (2)

The ambiguities and incorrect answers that lead to erroneous evidentiary procedures and conclusions are due to incomplete use of language, specifically in 
Mathematics in the lack of consistency of the initial hypotheses - terms with the properties of the extended body.

Namely, there are elements of the immersed body in another body which do not have a corresponding element in the extension thereof and the extension involves a restriction of the initial one. For example: when extending the body of fractional numbers and immersing it in the body of decimals $0.333333 \ldots$... this does not belong to real numbers and the fraction $1 / 3$ has no representation in the set of decimals (Kappos 1962, p. 58).

Theorem - Complement (1)

The remainder of the division of an integer polynomial $\mathrm{f}(\mathrm{x})$ with the binomial $(\mathrm{x}+\mathrm{c}) \neq 0 \rightarrow \mathrm{x} \neq-\mathrm{c}$ as divisor, is $\mathrm{f}(-\mathrm{c})$.

Respectively, in the ring of integers the $\mathrm{f}(-\mathrm{c})$ is the (partial?) remainder with the value of $\mathrm{x}+\mathrm{c} \in \mathrm{N}$, when $\mathrm{x} \neq-\mathrm{c}$.

\section{Proof}

Let $\mathrm{f}(\mathrm{x})=\sum_{\mathrm{i}=\mathbf{0}}^{\mathrm{v}} \boldsymbol{\alpha}_{\mathrm{i}} \chi^{\mathrm{i}}=\sum_{\mathrm{i}=\mathbf{0}}^{\mathbf{v}} \boldsymbol{\alpha}_{\mathbf{i}}(\chi+\mathbf{c}-\mathbf{c})^{\mathrm{i}}=$ $=\sum_{\mathbf{i}=\mathbf{0}}^{\mathbf{v}}\left\{\boldsymbol{\alpha}_{\mathbf{i}}\left[\sum_{\mathbf{k}=\mathbf{0}}^{\mathbf{i}}\left(\begin{array}{l}\mathbf{i} \\ \mathbf{k}\end{array}\right)(\chi+\mathbf{c})^{\mathbf{k}}(-\mathbf{c})^{\boldsymbol{v}-\mathbf{k}}\right]+\boldsymbol{\alpha}_{\mathbf{i}}(-\mathbf{c})^{\mathbf{i}}\right\}=$ $=\quad \sum_{\mathrm{i}=1}^{v}\left\{\alpha_{\mathrm{i}}(\chi+\mathrm{c}) \sum_{\mathrm{k}=1}^{\mathrm{i}}\left(\begin{array}{l}\mathrm{i} \\ \mathrm{k}\end{array}\right)(\chi+\mathrm{c})^{\mathrm{k}-1}(-\mathrm{c})^{v-\kappa}\right\}$ $+\sum_{\mathbf{i}=\mathbf{0}}^{\mathbf{v}} \boldsymbol{\alpha}_{\mathbf{i}}(-\mathbf{c})^{\mathbf{i}}=$

$$
=(\chi+c) \sum_{\mathbf{i}=1}^{v}\left\{\alpha_{\mathbf{i}} \sum_{\mathbf{k}=1}^{\mathbf{i}}\left(\begin{array}{l}
\mathbf{i} \\
\mathbf{k}
\end{array}\right)(\chi+\mathbf{c})^{\kappa-1}(-\mathbf{c})^{v-\kappa}\right\}+\mathrm{f}
$$
$(-\mathrm{c})$.

Because $f(-c)$ is constant is zero degree and because the

$$
(\chi+c) \sum_{i=1}^{v}\left\{\alpha_{i} \sum_{\kappa=1}^{i}\left(\begin{array}{l}
i \\
\kappa
\end{array}\right)(\chi+c)^{\kappa-1}(-c)^{\nu-\kappa}\right\} \text { is }
$$
divided by $(\chi+c)$ the balance (partial?) dividing by $(\chi$ $+c)$ is the remainder of $\mathrm{f}(-\mathrm{c})$ with a divider $(\chi+\mathrm{c})$

(In integer polynomials is the same $\mathrm{f}(-\mathrm{c})$, because it has a degree less than the degree $(\chi+c))$.

When there prices $\chi_{i}$ order of $\mathrm{f}$ $(\chi)$ $(\chi+c) \sum_{i=1}^{v}\left\{\alpha_{i} \sum_{\kappa=1}^{i}\left(\begin{array}{l}i \\ \kappa\end{array}\right)(\chi+c)^{\kappa-1}(-c)^{\nu-\kappa}\right\}=\mathrm{f}(-\mathrm{c})$ so $\mathrm{f}(-\mathrm{c})=\kappa(\chi+\mathrm{c}), \kappa \in \mathbf{N}$ to be an integer, then $\chi+\mathrm{c}$ divides $f(-c)$, thus dividing and $f(\chi)$ and the remainder is 0 .

Because $\mathrm{f}(-\mathrm{c})$ is a constant, it is zero degree and because

the $(\chi+c) \sum_{i=1}^{v}\left[\alpha_{i}\left\{\sum_{\kappa=1}^{i}\left(\begin{array}{l}i \\ \kappa\end{array}\right)(\chi+c)^{\kappa-1}(-c)^{i-\kappa}\right\}\right]$ divided by $(\chi+c)$, the partial remainder of division with $(\chi+c)$ $\neq 0$ is the remainder of $\mathrm{f}(-\mathrm{c})$ with divisor $(\chi+\mathrm{c})$.

(In integer polynomials it is the $\mathrm{f}(-\mathrm{c})$ itself, because it has a lesser degree than that of $(\chi+c)$ ).

Where there are values $\chi_{j}$ of $f(-c):(\chi+c)$ it is an integer, then $\chi+\mathrm{c}$ divides $\mathrm{f}(-\mathrm{c})$, thus also dividing $\mathrm{f}(\chi)$ and the remainder is $0\left\{\left(\chi_{\mathrm{i}}+\mathrm{c}\right)\right.$ factor of $\left.\mathrm{f}(-\mathrm{c})\right\}$.

Conclusion The quotient of the division $\mathrm{f}(\chi):(\chi+\mathrm{c})$ is $\sum_{\mathbf{i}=\mathbf{1}}^{v}\left\{\alpha_{\mathbf{i}} \sum_{\mathbf{k}=1}^{\mathbf{i}}\left(\begin{array}{l}\mathbf{i} \\ \mathbf{\kappa}\end{array}\right)(\chi+\mathbf{c})^{\boldsymbol{\kappa}-1}(-\mathbf{c})^{\boldsymbol{v}-\boldsymbol{\kappa}}\right\}$.

Up until now, the process of finding the remainder of $\mathrm{f}(\chi):(\chi+\mathrm{c})$ with replacement of $\chi$ with $\chi=-\mathrm{c}$ is at least incomplete

(it is valid with certainty (?), when $\delta(\chi)=\chi+\mathrm{c}=0$.)

TO SOLVE the problem:

To determine the remainder of division of integer polynomials (which by definition are an expression of the real problem function rule)

$\left(\chi^{6}+1\right):(\chi+\sqrt{10}), 1^{\text {st }} \chi \in \mathrm{Q}$ and $2^{\text {nd }} \chi \in R$,

(The coefficients of the terms of integer

polynomials $\mathrm{a}_{\mathrm{i}} \in \mathrm{Q}$ in general)

$1^{\text {st }}: v=f(-\sqrt{10})=1001$, larger than the remainder with a value of $\mathrm{x}$ in the interval

$-1004<\chi<996$.

$2^{\text {nd }}$ : When $\chi^{6}+1$ is defined in $\mathrm{N}$, then $\chi$ does not take the value of an irrational number.

New Theorem (2)

The trinomial $\chi^{2}+\beta \chi+\gamma$ takes square values when and only when there are two factors of the discriminant $\beta^{2}-4 \gamma$, supposing $\delta_{1}, \delta_{2}$ with $\delta_{2}-\delta_{1}$ being a multiple of 4 and $\delta_{2} \delta_{1}=\Delta$ or $\Delta-1=$ a multiple of 4 .

If $\Delta$ is a prime number, it is sufficient for $\Delta-1$ to be a multiple of 4 .

Proof.

$x^{2}+\beta x+\gamma=(x+\rho)^{2}(1) \rightarrow x^{2}+\beta x+\gamma=x^{2}$ $+2 \rho \chi+\rho^{2} \rightarrow(\beta-2 \rho) x=\rho^{2}-\gamma \rightarrow \chi=\frac{\rho^{2}-\gamma}{\beta-2 \rho} \rightarrow \rightarrow \chi=$ $\left(\rho^{2}-\gamma\right):(-2 \rho+\beta)=-\frac{1}{2} \rho-\frac{1}{4} \beta+\frac{\frac{1}{4} \beta^{2}-\gamma}{-2 \rho+\beta}=\frac{1}{4}\{-2 \rho-$ 
$\left.\beta+\frac{\beta^{2}-4 \gamma}{\beta-2 \rho}\right\}$. Since $\chi$ is an integer, $\beta^{2}-4 \gamma$ is divided by $\beta-2 \rho \rightarrow$ then $\beta-2 \rho=\delta_{i}$ is a factor of $\beta^{2}-4 \gamma .\left(\beta^{2}-4 \gamma=\right.$ discriminant of the trinomial)

If there is $\delta_{\mathrm{i}}$, we examine the divisor of $\beta^{2}-4 \gamma$ (if not, we examine the pair $1, \Delta$ ), we put $\beta-2 \rho=\delta_{j} \rightarrow \rho=$ $\frac{\beta-\delta_{i}}{2}(2)$

from $(2) \rightarrow \mathbf{X}=\frac{1}{4}\left\{-2 \frac{\beta-\delta_{\mathbf{j}}}{2}-\beta+\delta^{\prime}{ }_{j}\right\}=\frac{1}{4}\left\{\delta^{\prime}{ }_{j}+\right.$ $\boldsymbol{\delta j}-2 \boldsymbol{\beta}$ (3) with $\delta^{\prime} \mathrm{j}=\beta 2-4 \mathrm{c}: \delta \mathrm{j} . \rightarrow(4) \rightarrow \mathrm{x} 2+\beta \mathrm{x}+\gamma=$ $\left\{\frac{1}{4}\left(\delta_{j}^{\prime}+\delta_{j}-2 \beta\right)\right\}^{2}+\beta \frac{1}{4}\left(\delta_{j}^{\prime}+\delta_{j}-2 \beta\right)+\gamma=$ $=\frac{1}{4}\left(\delta_{j}^{\prime}+\delta_{\mathrm{j}}-2 \beta\right)\left\{\frac{1}{4}\left(\delta_{\mathrm{j}}^{\prime}+\delta_{\mathrm{j}}-2 \beta\right)+\beta\right\}+\gamma=$ $=\frac{1}{16}\left(\delta_{j}^{\prime}+\delta_{j}-2 \beta\right)\left\{\delta_{j}^{\prime}+\delta_{j}-2 \beta+4 \beta\right\}+\gamma=$ $=\frac{1}{16}\left(\delta_{j}^{\prime}+\delta_{j}-2 \beta\right)\left(\delta_{j}^{\prime}+\delta_{j}+2 \beta\right)+\gamma=$ $\frac{1}{16}\left\{\left(\delta_{j}^{\prime}+\delta_{j}\right)^{2}-4 \beta^{2}\right\}+\gamma$ Therefore, the value of the trinomial, where $\chi=\frac{1}{4}\left(\delta_{\mathrm{j}}+\delta_{\mathrm{i}}-2 \beta\right)$ is from (1), (2), (3) and (4)

$$
x^{2}+\beta \chi+\gamma=\left\{\frac{1}{4}\left(\delta_{j}^{\prime}+\delta_{j}-2 \beta\right)+\frac{\beta-\delta_{j}}{2}\right\}^{2}=
$$
$\frac{1}{16}\left(\delta_{j}^{\prime}-\delta_{j}\right)^{2}=\left(\frac{\delta_{j}^{\prime}-\delta_{j}}{4}\right)^{2}$ If $\Delta>0$, then or $\delta_{j}^{\prime}, \delta_{j}$ are omosemoi. $\rightarrow \delta_{j}^{\prime}-\delta_{j}= \pm|| \delta_{j}^{\prime}|-| \delta_{j}||$. So $\mathrm{f}(\chi)=$ $\left( \pm \frac{1}{4}|| \delta_{j}^{\prime}|-| \delta_{j}||\right)^{2}=\left(\frac{1}{4}\left(\left|\delta_{j}^{\prime}\right|-\left|\delta_{j}\right|\right)\right)^{2}$. If $\Delta<0$, then are $\boldsymbol{\delta}_{\boldsymbol{j}}^{\prime}, \boldsymbol{\delta}_{\boldsymbol{j}}$ eterosemoi $\rightarrow \rightarrow \boldsymbol{\delta}_{\boldsymbol{j}}^{\prime}-\boldsymbol{\delta}_{\boldsymbol{j}}= \pm\left|\boldsymbol{\delta}_{\boldsymbol{j}}^{\prime}\right|-$ $\left(\mp\left|\delta_{j}\right|\right)= \pm\left|\delta_{j}^{\prime}\right| \pm\left|\delta_{j}\right|= \pm\left(\left|\delta_{j}^{\prime}\right|+\left|\delta_{j}\right|\right)$.

So $\mathrm{f}(\chi)=\left( \pm \frac{1}{4}\left(\left|\delta_{j}^{\prime}\right|+\left|\delta_{j}\right|\right)\right)^{2}=\left(\frac{1}{4}\left(\left|\delta_{j}^{\prime}\right|+\left|\delta_{j}\right|\right)\right)^{2}$

Examble:

$\chi^{2}+5 \chi+1$

$\Delta=5^{2}-4.1=21 \rightarrow\left(\boldsymbol{\delta}^{\prime}{ }_{j}, \boldsymbol{\delta}_{\boldsymbol{j}}\right)=(3,7), \dot{\eta}(1,21), \dot{\eta}$ $(-3 .-7), \eta(-1,-21)$ when $\chi=\frac{1}{4}(7+3-2.5)=0$, then $\chi^{2}+5 \chi$ $+1=0+0+1=\frac{\mathbf{1}}{\mathbf{1 6}}(\mathbf{7}-\mathbf{3})^{2}=1^{2}$, when $\chi=\frac{\mathbf{1}}{\mathbf{4}}$

$(21+1-2.5)=3$, then $\chi^{2}+5 \chi+1=9+15+1=\frac{\mathbf{1}}{\mathbf{1 6}}(\mathbf{2 1}-\mathbf{1})^{2}$ $=5^{2}$ when $\chi=\frac{1}{4}(-7-3-2.5)=-5$, then $\chi^{2}+5 \chi+1=$ $25-25+1=\frac{1}{16}(-7+3)^{2}=1^{2}$ when $\chi=\frac{1}{4}(-21-1$ $-2.5)=-8$, then $\chi^{2}+5 \chi+1=64-40+1=25=$ $\frac{1}{16}((-21)-(-1))^{2}=5^{2}$

Examble

2) $\chi^{2}+\chi+3 \Delta=-11 \rightarrow\left(\boldsymbol{\delta}_{j}^{\prime}, \boldsymbol{\delta}_{j}\right)=(1,-11)$, ๆ́ $(-1,11)$. When $\chi=\frac{1}{4}(1-11-2.1)=-3 \rightarrow \chi^{2}+\chi+3=9$ $=\frac{1}{16}(1+11)^{2}=3^{2}$ When $\chi=\frac{1}{4}(-1+11-2.1)=$ $2 \rightarrow \chi^{2}+\chi+3=9=\frac{1}{16}(-1-11)^{2}=3^{2}$

Examble 3) $\mathrm{f}(\mathrm{x})=\boldsymbol{x}^{2}+\mathbf{2 6} \boldsymbol{x}+\mathbf{9}$

$\Delta=26^{2}-4.9=676-36=640=4^{3} .2 .5$

$\left(\delta_{j}^{\prime}, \delta_{j}\right) \delta_{j}^{\prime}-\delta_{j} \chi=\frac{1}{4}\left(\delta_{j}^{\prime}+\delta_{j}-2 \beta\right)$

(4, $4^{2}$ $.2 .5)$ $4(1-40)=4 .(-39)$ $\chi=\frac{1}{4}\left(4+4^{2} \cdot 2 \cdot 5-2 \cdot 26\right)=28 \quad f(\chi)=2^{2}+26 \cdot 28+9=$ $=1521=39^{2}$

$\left(\mathbf{4}^{2}, \mathbf{4} . \mathbf{2} .5\right) \quad 4(4-10)=4(-6) \quad \chi=\frac{\mathbf{1}}{\mathbf{4}}\left(\mathbf{4}^{2}+4.2 .5-2.26\right)=1$ $f(\chi)=\mathbf{1}^{2}+26.1+9=36=6^{2}$

$\left(4.2, \mathbf{4}^{2} .5\right) \quad 4(2-20)=4 .(-18) \quad \chi=\frac{\mathbf{1}}{\mathbf{4}}\left(4 \cdot 2+\mathbf{4}^{2} \cdot \mathbf{5}-2 \cdot 26\right)=9$ $\mathrm{f}(\chi)=\mathbf{9}^{2}+26.9+9=324=\mathbf{1 8}^{2}$

$\left(\mathbf{4}^{2} .2,4.5\right) \quad 4(8-5)=4.3 \quad \chi=\frac{1}{\mathbf{4}}\left(\mathbf{4}^{2} \cdot \mathbf{2}+4.5-2.26\right)=0$ $\mathrm{f}(\chi)=\mathbf{0}^{2}+26.0+9=9=\mathbf{3}^{2}$

$\left(4.5, \mathbf{4}^{2} .2\right) \quad 4(5-8)=4(-3) \quad \chi=\frac{\mathbf{1}}{\mathbf{4}}\left(4.5+\mathbf{4}^{2} \cdot 2-2.26\right)=0$ $\mathrm{f}(\chi)=\mathbf{0}^{2}+26.0+9=9=\mathbf{3}^{2}$

$\left(\mathbf{4}^{2} \cdot \mathbf{5}, 4.2\right) \quad 4(20-2)=4.18 \quad \chi=\frac{1}{\mathbf{4}}\left(\mathbf{4}^{2} \cdot 5+4 \cdot 2-2 \cdot 26\right)=9$ 
$f(\chi)=\mathbf{9}^{2}+26.0+9=324=\mathbf{1 8}^{2}$

$$
\left(4.2 .5, \mathbf{4}^{2}\right) \quad 4(10-4)=4.6 \quad \chi=\frac{1}{\mathbf{4}}\left(4.2 .5+\mathbf{4}^{2}-2.26\right)=1
$$

$f(\chi)=\mathbf{1}^{2}+26.1+9=36=6^{2}$

$$
\left(\mathbf{4}^{2} \cdot 2.5,4\right) \quad 4(40-1)=4.39 \quad \chi=\frac{1}{4}\left(\mathbf{4}^{2} \cdot 2.5+4-2.26\right)=28
$$

$\mathrm{f}(\chi)=\mathbf{2 8}^{2}+26.28+9=1521=\mathbf{3 9}^{2}$ is divisors of $\Delta$ $\left(-\boldsymbol{\delta}_{\boldsymbol{j}}^{\prime},-\boldsymbol{\delta}_{\boldsymbol{j}}\right)$ which $-\boldsymbol{\delta}_{\boldsymbol{j}}^{\prime}-\left(-\boldsymbol{\delta}_{\boldsymbol{j}}\right)=-\left(\boldsymbol{\delta}_{\boldsymbol{j}}^{\prime}-\boldsymbol{\delta}_{\boldsymbol{j}}\right)=4 .(\mathrm{)})$

that yields the same perfect squares various values of $\mathrm{x}$ from

$\{-54,-27,-35,-26,-26,-35,-27,-54\}$

Therefore, a necessary and sufficient condition for the numerical value of the trinomial being a perfect square integer is to have two divisors of the discriminant, namely $\delta_{2}$ and $\delta_{1}$, with $\delta_{2}-\delta_{1}$ being a multiple of 4 and $\delta_{2} \delta_{1}=\Delta$.

The finding of pairs, when the product $\delta_{j}^{\prime} \delta_{j} \Delta$ is not divisible by 4 , is arrived at through the solution of the family of equations $\chi^{2} \pm \boldsymbol{4}^{v} \kappa \chi-\Delta=0$, with $\boldsymbol{\delta}_{\boldsymbol{i}}^{\prime}>\boldsymbol{\delta}_{\boldsymbol{i}}$

and $4<4^{v} \kappa<|\Delta| \rightarrow \quad v<\frac{\log |\Delta|}{\log 4}$ and

$\frac{2 \sqrt{|\Delta|}}{4^{v}}<\kappa<\frac{|\Delta|}{4^{v}}$. We seek the values of $v, \mathrm{k}$ so that $\Delta^{\prime}=\mathbf{4}^{2 v}+4 \Delta$ or $\mathbf{4}^{2 v-1}+\Delta$ to be square values.

If one of the $\boldsymbol{\delta}_{\boldsymbol{j}}^{\prime}, \boldsymbol{\delta}_{\boldsymbol{j}}$ is divisible by 4 or $\Delta$ is divisible by $\mathbf{4}^{v}, v \geq 2$, then the other two are also divisible by 4 and the number of pairs is $(v-1)$ $\prod_{i=1}^{\boldsymbol{k}}\left(\boldsymbol{v}_{\boldsymbol{i}}+\mathbf{1}\right), \boldsymbol{v}_{\boldsymbol{i}}$ is the exponent of coefficient $\boldsymbol{\mu}_{\boldsymbol{i}}$ of $\Delta=\mathbf{4}^{v} \prod_{i+1}^{\kappa} \boldsymbol{\mu}_{i}^{v_{i}}$. If $\Delta=0$, then for each number $\chi=\boldsymbol{\delta}_{\boldsymbol{j}}$ there is a pair $\left(\boldsymbol{\delta}_{\boldsymbol{j}}, \boldsymbol{\delta}_{\boldsymbol{j}}+4 \lambda\right)$, which satisfies the conditions of theorem (the difference is divisible by 4 and, $\boldsymbol{\delta}_{\boldsymbol{j}}, \boldsymbol{\delta}_{\boldsymbol{j}}+4 \lambda$ divides $\Delta=0$ ). Consequently, the trinomial is a perfect square of a polynomial.

A language (and its configuration in mathematical language) that depicts the truth so that it can be used by computers and be posted on the Internet is sought by the global scientific community. Consequently, in addition to the scientific problem itself, whose development is remarkable, three additional substantial problems have emerged: 1) The certification of truth. 2) The exploitation of truth and 3) Monitoring the practice of truth (Werner Heisenberg: Encounters with Einstein).

It is imperative that knowledge bears the hallmarks of truth and useful and safe use. Of course, the development of computers is necessary and advantageous; however, their use in the educational and research process is no substitute for the ability of human thought, which can 'play' and 'move' over the desktop, transferring and modifying procedures and observations from experiences of human action in general into scientific processes, looking for links in the 'mathematical horizon', creating and demonstrating entries. We should stress the conclusions of Bloom's taxonomy (Volume II) for the emotional world in which the user participates. Consequently, to ignore and alter appropriate and established knowledge and procedure may not be considered challenging, but it is NEW knowledge, a NEW take on an existing situation. Knowledge is simplified, and becomes useful and understandable.

Example-Transformation of $b^{n}+c^{n}=a^{n}$ (L.T.F.), when $\mathrm{n}$ divides $\mathrm{abc}$ in a quadratic equation and solution.

If $\alpha \beta \gamma$ is divisible by $v$ in Fermat's last theorem, there are no $\alpha, \beta, \gamma$ integers, so that $\beta^{v}+\gamma^{v}=\alpha^{v}$

Proof

Without limitation of the proof, we accept as proven that:

1) $\alpha, \beta, \gamma, v$ are positive integers, per two are relatively prime numbers and $v$ is a prime number, which may take the form $v=6 \kappa \pm 1$.

(Stamatopoulos

Spyros ICM2010HYNDERABATT)

2) $\beta+\gamma=v^{v-1} \alpha_{11}^{v}, \sum_{i=1}^{v}(-1)^{\nu-i} \beta^{v-i} \gamma^{i-1}=v \alpha_{12}^{v}, \alpha=$ $v \alpha_{1}, \alpha_{1}=\alpha_{11} \alpha_{12}$ and $\operatorname{gcd}\left(\alpha_{11}, \alpha_{12}\right)=1$

A) $v=6 \mathrm{k}+1$ from $\beta^{v}+\gamma^{v}=v^{v} \alpha_{11}^{v} \alpha_{12}^{v} \rightarrow \beta^{6 \kappa+1}+$ $\gamma^{6 \kappa+1}=v^{v} \alpha_{11}^{v} \alpha_{12}^{v} \rightarrow \rightarrow \beta \beta^{6 \kappa}+\gamma^{6 \kappa+1}=v^{v} \alpha_{11}^{v} \alpha_{12}^{v} \rightarrow$ $\left(v^{v-1} \alpha_{11}^{v}-\gamma\right) \beta^{6 \kappa}+\gamma^{6 \kappa+1}=v^{v} \alpha_{11}^{v} \alpha_{12}^{v} \rightarrow$ $\gamma\left(\gamma^{6 \kappa}-\beta^{6 \kappa}\right)=v^{v-1} \alpha_{11}^{v}\left(v \alpha_{12}^{v}-\beta^{6 \kappa}\right) \rightarrow$ 


$$
\begin{aligned}
& \gamma\left(\beta^{2 \kappa}-\gamma^{2 \kappa}\right)\left(\beta^{4 \kappa}+\gamma^{2 \kappa} \beta^{2 \kappa}+\gamma^{4 \kappa}\right) \\
& =v^{v-1} \alpha_{11}^{v}\left(\beta^{6 \kappa}-v \alpha_{12}^{v}\right)
\end{aligned}
$$

Because $\beta \neq \gamma \rightarrow \beta^{2 \kappa}-\gamma^{2 \kappa} \neq 0$, so the first member is divisible by $\gamma\left(\beta^{2 \kappa}-\gamma^{2 \kappa}\right)$ and thus the second State shall be divided by $\gamma\left(\beta^{2 \kappa}-\gamma^{2 \kappa}\right)$. Let

$$
v^{v-1} \alpha_{11}^{v}\left(\beta^{6 \kappa}-v \alpha_{12}^{v}\right)=\gamma\left(\beta^{2 \kappa}-\gamma^{2 \kappa}\right) p \text {. }
$$

Then the equation (5) or

$$
\begin{aligned}
\gamma\left(\beta^{2 \kappa}-\gamma^{2 \kappa}\right) \beta^{4 \kappa} & +\gamma\left(\beta^{2 \kappa}-\gamma^{2 \kappa}\right) \gamma^{2 \kappa} \beta^{2 \kappa} \\
& +\gamma\left(\beta^{2 \kappa}-\gamma^{2 \kappa}\right) \gamma^{4 \kappa} \\
& -v^{v-1} \alpha_{11}^{v}\left(\beta^{6 \kappa}-v \alpha_{12}^{v}\right)=0
\end{aligned}
$$

is writing

$$
\begin{array}{r}
\gamma\left(\beta^{2 \kappa}-\gamma^{2 \kappa}\right)\left(\beta^{4 \kappa}+\gamma^{2 \kappa} \gamma^{2 \kappa}+\gamma^{4 \kappa}\right) \\
=\gamma\left(\beta^{2 \kappa}-\gamma^{2 \kappa}\right) p
\end{array}
$$

or

$$
\begin{aligned}
\gamma\left(\beta^{2 \kappa}-\gamma^{2 \kappa}\right) \beta^{4 \kappa} & +\gamma\left(\beta^{2 \kappa}-\gamma^{2 \kappa}\right) \gamma^{2 \kappa} \beta^{2 \kappa} \\
& +\gamma\left(\beta^{2 \kappa}-\gamma^{2 \kappa}\right)\left(\gamma^{4 \kappa}-p\right)=0 .
\end{aligned}
$$

So is of the form $\mathrm{KX}^{2}+\mathrm{KBX}+\mathrm{K} \Gamma=0$, where $\mathrm{X}=\beta^{2 \kappa}$, $\mathrm{K}=\gamma\left(\beta^{2 \kappa}-\gamma^{2 \kappa}\right)$ and $\Gamma=\gamma^{4 \kappa}-\mathrm{p}$, or (7) $\mathrm{K}\left(\mathrm{X}^{2}+\mathrm{BX}+\right.$ $\Gamma)=0$.Because $\kappa \neq 0$ the $X$ is a radical of the equation $\mathrm{X}^{2}+\mathrm{BX}+\Gamma=0$. (8) Because $\mathrm{X}=\beta^{2 \kappa}$ is an integer the discriminan $\Delta=\mathrm{B}^{2}-4 \Gamma$ must be a perfect square.

Lemma:

The number $\mathrm{Z}=(\mathrm{KB})^{2}-4 \mathrm{KK} \Gamma=\mathrm{K}^{2}\left(\mathrm{~B}^{2}-\right.$ $4 \Gamma=\mathrm{K} 2 \Delta$ in the equation (7) is perfect square, when and only when the $\Delta=B^{2}-4 \Gamma$, in equation (8) is a perfect square.

Proof. If $\mathrm{Z}=\zeta^{2} \rightarrow \Delta=\frac{\zeta^{2}}{\mathrm{~K}^{2}}=\left(\frac{\zeta}{\mathrm{K}}\right)^{2}$. If $\Delta=\delta^{2} \rightarrow$ $\mathrm{Z}=\mathrm{K}^{2} \delta^{2}=(\mathrm{K} \delta)^{2}$. From equation $\rightarrow Z=\gamma^{2} \gamma^{4 \kappa}\left(\beta^{2 \kappa}-\gamma^{2 \kappa}\right)^{2}-4 \quad \gamma^{2} \gamma^{4 \kappa}\left(\beta^{2 \kappa}-\gamma^{2 \kappa}\right)^{2}+$ $+4 \gamma\left(\beta^{2 \kappa}-\gamma^{2 \kappa}\right) v^{v-1} \alpha_{11}^{v}\left(\beta^{6 \kappa}-v \alpha_{12}^{v}\right)=$

$$
=-3 \gamma^{4 \kappa+2}\left(\beta^{2 \kappa}-\gamma^{2 \kappa}\right)^{2}+4 \gamma
$$

$$
\left(\beta^{2 \kappa}-\gamma^{2 \kappa}\right) v^{v-1} \alpha_{11}^{v}\left(\beta^{6 \kappa}-v \alpha_{12}^{v}\right)
$$

$\rightarrow \Delta=\frac{\mathrm{Z}}{\mathrm{K}^{2}}=-3 \gamma^{4 \kappa}+\frac{4 v^{v-1} \alpha_{11}^{v}\left(\beta^{6 \kappa}-v \alpha_{12}^{v}\right)}{\gamma\left(\beta^{2 \kappa}-\gamma^{2 \kappa}\right)}$ The denominator factor is the $\beta-\gamma$, so the numerator divided by $\beta-\gamma$.

Is $4 v^{v-1} \alpha_{11}^{v}=4(\beta+\gamma)=4(\beta-\gamma)+8 \gamma$, so the $\beta-\gamma$ does not divide $4 v^{v-1} \alpha_{11}^{v}$ because is not divides the $8 \gamma$. So the $\beta-\gamma$ divides $\beta^{6 \kappa}-v \alpha_{12}^{v}$. The rest of the numerator with $\beta-\gamma$

$$
\begin{aligned}
& \text { Is } v=\gamma^{6 \kappa}-v(\gamma, \gamma)_{12}^{v} . \\
& \text { If } v=\gamma^{v-1}-v \alpha(\gamma, \gamma)_{12}^{v}=0 \text { then } \gamma^{v-1}=v \alpha(\gamma, \gamma)_{12}^{v} \text {, }
\end{aligned}
$$
which is impossibility because $v$ divided $\gamma$. This is contrary to the restriction $\operatorname{gcd}(\mathrm{c}, \mathrm{n})=1$. Therefore $\mathrm{D}$ does not take integer values (does not have divisors, etc. theorem.) and therefore it is not a perfect square, and $b$ is not an integer.

If $\beta-\gamma<v$, the algebraic remainder $v=\gamma^{6 \kappa}-v \alpha(\gamma, \gamma)_{12}^{v}$ may take values greater than $\beta-\gamma$ for some values of $\beta, \gamma$.

If $v=\gamma^{6 \kappa}-v \alpha(\gamma, \gamma)_{12}^{v}>\beta-\gamma$ then

$$
\alpha_{2}^{v}=\sum_{\mathrm{i}=1}^{v}(-1)^{\mathrm{i}-1} \beta^{\nu-\mathrm{i}} \gamma^{\mathrm{i}-1}=(\beta-\gamma) \sum_{\mathrm{i}=1}^{\frac{v-1}{2}} \beta^{\nu-2 \mathrm{i}} \gamma^{2 \mathrm{i}-2}
$$

$+\gamma^{v-1}$, therefore

$$
\begin{aligned}
& v_{\alpha_{2}^{v}:(\beta-\gamma)}=\gamma^{v-1} \rightarrow\left(v_{\alpha_{2}:(\beta-\gamma)}\right)^{v}=\gamma^{v-1} \rightarrow \\
& v_{\alpha_{2}:(\beta-\gamma)} \sqrt[v-1]{v_{\alpha_{2}:(\beta-\gamma)}}=\gamma .(9) \\
& \text { Because } v_{\alpha_{2}:(\beta-\gamma)}<\beta-\gamma \leq v-1 \rightarrow \\
& v-1 \sqrt{v_{\alpha_{2}:(\beta-\gamma)}} \text { is not integer when } v_{\alpha_{2}:(\beta-\gamma)} \neq 1 \\
& v_{\alpha_{2}:(\beta-\gamma)}=1 \text { From (9) } \gamma=1 \text {. Because } \alpha_{2}=v \alpha_{12} \rightarrow \\
& v_{v:(\beta-\gamma)}=v_{\alpha_{12}:(\beta-\gamma)}=1 \\
& \text { From } \beta+\gamma=v^{v-1} \alpha_{11}^{v} \rightarrow v_{\beta:(\beta-\gamma)}+v_{\gamma:(\beta-\gamma)}= \\
& v_{v:(\beta-\gamma)}^{v-1} v_{\alpha_{11}^{v}:(\beta-\gamma)} \rightarrow \\
& \rightarrow v_{\beta:(\beta-\gamma)}+1=\left(v_{\alpha_{11}:(\beta-\gamma)}\right)^{v} \text { (10). Because }
\end{aligned}
$$
$v_{\beta:(\beta-\gamma)}<\beta-\gamma<v \rightarrow\left[v_{\alpha_{11}:(\beta-\gamma)}\right]^{v}=v_{\beta:(\beta-\gamma)}+1<v$ $\rightarrow v_{\alpha_{11}:(\beta-\gamma)}=1$.

Therefore from (10), $v_{\beta:(\beta-\gamma)}+1=1 \rightarrow v_{\beta:(\beta-\gamma)}=$ 0 so the $\beta$ divided by $\beta-\gamma$ which is impossibility because $\operatorname{gcd}(\beta,(\beta-\gamma)))=1$.

(The $\alpha_{2}^{v}$ are homogeneous polynomial therefore the $\gamma^{v}$ is factor of $v[\alpha(\gamma, \gamma)]_{12}^{v} \rightarrow \rightarrow v[\alpha(\gamma, \gamma)]_{12}^{v}=$ $v \gamma^{v} \rho$. From (9) $\rightarrow v=\gamma^{v-1}-v[\alpha(\gamma, \gamma)]_{12}^{v}=\gamma^{6 \kappa}{ }_{-}$ $v \gamma^{6 \kappa+1} \rho=\gamma^{6 \kappa}(1-v \gamma \rho)$

1) If $\gamma^{6 \kappa}(1-v \gamma \rho)<\beta-\gamma$ then $v=\gamma^{6 \kappa}(1-v \gamma \rho) \neq 0$

2) If $\gamma^{6 \kappa}(1-v \gamma \rho)>\beta-\gamma$ and this divided by $(\beta-\gamma)$, then the $(1--v \gamma \rho)$ divided by

$\beta-\gamma$, because $\operatorname{gcd}(\gamma, \beta-\gamma)=1)$. 


$$
\begin{aligned}
& \text { Therefore } v_{[(1-v \gamma \rho)]:(\beta-\gamma)}=0 \rightarrow 1 \quad \\
& v_{v:(\beta-\gamma)} v_{\gamma:(\beta-\gamma)} v_{\rho:(\beta-\gamma)}=0 \rightarrow \rightarrow v_{v:(\beta-\gamma)}=v_{\gamma:(\beta-\gamma)}= \\
& v_{\rho:(\beta-\gamma)}=1 \text { ect). } \\
& \quad \text { If } v=6 \kappa-1 \rightarrow \beta^{6 \kappa-1}+\gamma^{6 \kappa-1}=v^{v} \alpha_{1}^{v} \rightarrow \gamma \beta^{6 \kappa-1}+ \\
& \gamma^{6 \kappa}=\gamma v^{v} \alpha_{1}^{v} \rightarrow \rightarrow\left(v^{v-1} \alpha_{11}^{v}-\beta\right) \beta^{6 \kappa-1}+\gamma^{6 \kappa}=\gamma v^{v} \alpha_{1}^{v} \\
& \rightarrow \text { ect. } \\
& \quad \text { If } \quad \beta=v \beta_{1} \rightarrow \alpha^{v}-\gamma^{v}=\beta^{v} \rightarrow \\
& (\alpha-\gamma) \sum_{i=1}^{v} \alpha^{v-i} \gamma^{i-1}=v^{v} \beta_{1}^{v} \rightarrow \alpha-\gamma=v^{v-1} \beta_{11}^{v} \text { and } \rightarrow \\
& \quad \sum_{i=1}^{v} \alpha^{v-i} \gamma^{i-1}=v \beta_{12}^{v} \rightarrow \text { ect. }
\end{aligned}
$$

I believe that, in addition to what is available today, there remains a long way to go and a lot of effort to be made on the basis of the scientific ideal, in terms of richness, variety and access with free personal choice, to create an ideal environment for creative work.

\section{References}

[1] T. N. Kazantzis, Number theory

[2] Robert B. Ash Algebra p13,Capter2
[3] Philip A. Schmidt, From Arithmetic to Algebra, p.6

[4] D. Kappos. Calculus A, p. 58

[5] Spyros K. Kanellidis, Set Theory

[6] John B. Fraleigh, Introduction to Algebra,

[7] Spyros P. Stamatopoulos, IMC 2010

[8] Legh Wilber Reid, The Elements of Theory of Algebraic Numbers

[9] V. L. Smirnov, Robert B. Ash. The basic Graduate Year

[10] Lorin W. Anderson 2000, Assessing Affective Characteristics in the Schools Krathwohl D. R. 2000 (Alex. Lambraki-Panagou), Taxonomy of Educational Objectives, Bloom Volume II

[11] Carl M. Bender and Steven A. Orszag, Advanced Mathematical Methods for Scientists and Engineers

[12] Ilias V. Ntzioras, $2^{\text {nd }}$ Lyceum Year Algebra

[13] Werner Heisenberg, Encounters with Einstein

[14] Sources, Presentations

[15] GOOGL: WIKIA, GOOGL gr. Doxiadis - Filippou, Mathematical Horizon,

[16] Hellenic Mathematical Society of Patras: Lecture by Professor Evgenia Koleza, University of Patras on $18 / 05 / 15$ 